\title{
The Effects of Bariatric Procedures on Bowel Habit
}

\author{
Sorena Afshar ${ }^{1,2}$ (D) - Seamus B. Kelly ${ }^{1} \cdot$ Keith Seymour $^{1} \cdot$ Sean Woodcock $^{1}$. \\ Anke-Dorothee Werner ${ }^{2} \cdot$ John C. Mathers ${ }^{2}$
}

Published online: 19 February 2016

(C) The Author(s) 2016. This article is published with open access at Springerlink.com

\begin{abstract}
Background Bariatric procedures are increasingly being used to combat the rising obesity epidemic. The aim of this study was to assess the effect of these interventions on bowel habit. Methods We recruited obese adults listed for a bariatric procedure. Demographic data, medical history, medications and anthropometric measurements were recorded. Bowel habit was characterized using a 7-day Bristol Stool Form Scale (BSFS) diary. A validated food frequency questionnaire (FFQ) was used to assess diet.

Results Twenty-six patients were assessed pre-operatively and at a median of 6.4 months post-operatively. Nineteen had a Roux-en-Y gastric bypass (RYGB), five had a sleeve gastrectomy (SG) and two had an intra-gastric balloon (IGB) with median percentage excess weight loss (\% EWL) of 67.9, 52.4 and $31.3 \%$, respectively. Dietary fibre intake decreased from $24.4( \pm 12.1) \mathrm{g} /$ day pre-operatively to $17.5( \pm 7.3) \mathrm{g} /$ day post-operatively $(P=0.008)$. Frequency of bowel motions decreased from $8.6( \pm 3.5)$ to $5.7( \pm 3.5)$ motions/week $(P=0.001)$. Mean usual BSFS score decreased (towards firmer stool) from $4.1( \pm 1.3)$ pre-operatively to $3.1( \pm 1.9)$ post-operatively $(P=0.016)$. Constipation increased from 8 to $27 \%$, but this did not reach statistical significance $(P=0.125)$.

Conclusions Constipation is a common problem after bariatric surgery. The decrease in bowel motion frequency and
\end{abstract}

Sorena Afshar

s.afshar@doctors.org.uk

1 Northumbria Healthcare NHS Foundation Trust, North Shields, Tyne and Wear, UK

2 Newcastle University, Newcastle-upon-Tyne, Tyne and Wear, UK change towards firmer stools suggest prolonged intestinal transit time after bariatric procedures. Reduction in dietary fibre intake is likely to be a contributory factor.

Keywords Bariatric surgery - Bowel habit - Gut transit . Bristol stool form

\section{Introduction}

Worldwide prevalence of obesity is increasing. Obesity is a major risk factor for common chronic diseases, including diabetes, cardiovascular diseases and cancer [1]. Whilst lifestyle-based interventions are the backbone of most public health strategies to prevent and treat obesity, bariatric procedures are increasingly being used to combat this rising epidemic. These interventions have significant benefits in terms of achieving sustained weight loss and improvement of obesity-related comorbidities [2]. However, disordered bowel habit appears to be an associated problem in at least a subset of patients [3], and this can have a significant negative impact on the patient's quality of life $[4,5]$. Obesity itself is associated with disordered bowel habit with most studies showing increased prevalence of diarrhoea, but not constipation in obese patients $[3,6]$. This increased prevalence of diarrhoea is likely to be multifactorial and is likely to be related to diet. One hypothesis is that high intake of sugars and other products in excess of absorption capability leads to an osmotic diarrhoea [1].

The effects of specific bariatric procedures on bowel habit are not well defined. Some reports have suggested that the Roux-en-Y gastric bypass (RYGB) and biliopancreatic diversion (BPD) result in diarrhoea [7, 8]. In contrast, others have reported improvement in symptoms of loose stools after RYGB [9]. Most reports associate the adjustable gastric band 
(AGB) and sleeve gastrectomy (SG) with constipation [7, 8]. These inconsistencies may in part be due to the variety of validated and non-validated assessment tools used [3].

Bariatric procedures are likely to have procedure-specific effects on dietary intake as well as bowel habit. Therefore, to interpret the effects of such interventions on bowel habit, it is important to have longitudinal studies using validated assessment tools and robust information on dietary intake. There is a lack of such studies in the literature.

Therefore, as part of the observational study Biomarkers of Colorectal cancer After Bariatric Surgery (BOCABS study ISRCTN95459522), we assessed participants' dietary intake and bowel habits before and after bariatric procedures.

\section{Methods}

We recruited obese adults listed for a bariatric procedure at a single centre (North Tyneside General Hospital, North Shields, UK). Exclusion criteria were age $<18$ or $>65$ years, inflammatory bowel disease, previous weight loss surgery, previous colorectal resection, painful anorectal pathology (including anal fissure and symptomatic haemorrhoids) and pregnancy. Informed written consent was obtained from all individual participants included in the study.

Demographic data including age, sex, medical history and current medications were recorded for all participants. Anthropometric measurements including height, weight and waist and hip circumference were made using a standardised protocol by a single observer (SA). Percentage body fat was estimated using a bioimpedance device (Tanita TBF-300MA Body composition analyser). Data was collected preoperatively and repeated at 6 months post-operatively. All participants underwent digital rectal and rigid sigmoidoscopic examination to $15 \mathrm{~cm}$ from anal verge at each time point. Significant macroscopic abnormalities including malignancy, polyps $>1 \mathrm{~cm}$ and proctitis were planned for exclusion. A single random rectal biopsy, taken at each examination, was sent to a histopathologist for examination to exclude microscopic colitis.

\section{Bariatric Procedures}

The RYGB involved laparoscopic formation of a 50-ml gastric pouch with a $100-150-\mathrm{cm}$ alimentary limb and $60-75-\mathrm{cm}$ biliopancreatic limb. Alimentary limb length was determined by the patients' BMI and comorbidities $(150 \mathrm{~cm}$ for BMI $>50 \mathrm{~kg} / \mathrm{m}^{2}$ or in the presence of type 2 diabetes mellitus and $100 \mathrm{~cm}$ otherwise). Biliopancreatic limb length was chosen based on surgeon preference. The SG involved laparoscopic resection of the greater curvature of the stomach over a 34-36 F bougie. The intra-gastric balloon (IGB) was placed endoscopically (Allergen ${ }^{\circledR}$ ) and filled with 500-700 saline mixed with $10 \mathrm{ml}$ of methylene blue.

\section{Bowel Habit}

Bowel habit was characterized using a 7-day Bristol Stool Form Scale (BSFS) diary. This tool has been validated and has been shown to correlate with whole gut transit time (WGTT) [10-12]. Usual BSFS score was defined as the most commonly reported score during the diary period (mode). We defined constipation as fewer than three bowel motions per week with lumpy or hard stools (BSFS 1 or 2 ) in at least $25 \%$ of bowel motions recorded during the 7-day diary. Diarrhoea was defined as loose (mushy) or watery stools (BSFS 6 or 7) occurring in at least $75 \%$ of stools. These definitions are in keeping with the Rome III criteria for diagnosis of functional gastrointestinal disorders [13]. Ambiguous diary entries were clarified by contacting the participants.

\section{Dietary Assessment}

A validated food frequency questionnaire (FFQ) used in the European Prospective Investigation into Cancer and Nutrition (EPIC) study [14] was used to estimate the participant's usual dietary intake. The FFQ was modified to assess intake over the previous 3 months and not the previous year as per original questionnaire, and tailored slightly to include foods eaten commonly in the north-east of England.

\section{Statistical Analysis}

Data was collected in paper form and transferred to a studyspecific database. Statistical analysis was carried out using SPSS software (Version 22.0 for Windows, SPSS, Chicago, USA). The Shapiro-Wilk test was used to test for normality of distribution of variables. Data is reported as mean \pm standard deviations or median and interquartile range (IQR) as appropriate. Wilcoxon signed ranks, McNemar and paired sample $t$ tests were used as appropriate. Cross-tabulation was carried out using exact tests (Monte-Carlo simulation). ANOVA or Kruskal-Wallis tests were used to compare the different procedure groups as appropriate. Spearman's correlation coefficient was used to assess the strength of association between variables. Statistical significance was set at $P<0.05$.

\section{Results}

Thirty-eight candidates for a bariatric procedure were recruited; $32(84.2 \%)$ completed a BSFS diary at a median of 33 days pre-operatively (IQR 37.5).

Twenty-six out of the 32 (76.5\%) also completed a repeat diary at a median follow-up of 6.4 months (IQR 1.1) after the bariatric procedure. Therefore, 26 participants with paired data were suitable for inclusion. Participants had all successfully 
completed a 12-week weight management programme and achieved at least $5 \%$ body weight loss prior to enrolment.

Table 1 shows the characteristics of the study participants. There was no statistically significant difference in age and sex distribution between the treatment groups $(P=0.809$ and $P=0.260$, respectively). RYGB patients achieved the most weight loss, followed by SG and IGB with percentage excess weight loss (\% EWL-calculated based on ideal body weight of BMI $25 \mathrm{~kg} / \mathrm{m}^{2}$ ) of $67.9,52.4$ and $31.3 \%$, respectively $(P=0.043)$. There were no cases of significant anorectal pathology or microscopic colitis.

\section{Dietary Intake}

Table 2 shows intake of relevant dietary factors pre- and postoperatively. As expected, daily energy intake decreased $(P=0.023)$. There is also a statistically significant difference in fibre intake, decreasing from $24.4( \pm 12.1) \mathrm{g} /$ day preoperatively to $17.5( \pm 7.3) \mathrm{g}$ /day post-operatively $(P=0.008)$. Water, calcium and fat intake did not change significantly. Mean fibre intake was below the newly published Scientific Advisory Committee on Nutrition (SACN) recommended daily intake of $30 \mathrm{~g} /$ day at both the pre- and post-operative stage [15]. Only $38 \%$ (10 out of 26) and $15 \%$ (4 out of 26) of participants met this recommended intake level at the pre- and post-operative stage, respectively. This pattern was similar for all treatment groups.

\section{Frequency of Bowel Motions}

Interindividual variation in bowel motion frequency was very large pre- and post-operatively (Fig. 1). At 6.4 months after surgery, mean frequency of recorded bowel motions decreased from $8.5( \pm 3.7)$ to $5.7( \pm 3.3)$ motions/week $(P=0.001)$. This change in frequency of bowel motions was not influenced by the bariatric procedure performed $(P=0.149)$. There was no significant correlation between any of the anthropometric measures or dietary factors and the change in frequency of bowel motions. There was no significant correlation between the change in frequency of bowel motions and alimentary or biliopancreatic limb length (Spearman correlation coefficients; $r=0.184, P=0.480$ and $r=-0.168, P=0.520$, respectively. Data from 14 out of 19 RYGB cases; others missing data).

\section{Stool Form}

Table 3 shows the distribution of stool forms estimated using the usual BSFS pre- and post-operatively. Data from a large
Table 1 Participant characteristics

\begin{tabular}{|c|c|c|c|c|}
\hline & \multicolumn{3}{|c|}{ Surgical procedure } & \multirow{3}{*}{$P$ value } \\
\hline & RYGB & $\mathrm{SG}$ & IGB & \\
\hline & $N=19$ & $N=5$ & $N=2$ & \\
\hline Age (years)-median (IQR) & $49.0(7.8)$ & $44.6(26.8)$ & 49.1 & 0.809 \\
\hline \multicolumn{5}{|l|}{ Sex } \\
\hline Female (\%) & 84.2 & 80 & 50 & \multirow[t]{2}{*}{0.260} \\
\hline Male (\%) & 15.8 & 20 & 50 & \\
\hline \multicolumn{5}{|l|}{ Comorbidities $N(\%)$} \\
\hline Type II DM & $5(26)$ & $1(20)$ & $1(50)$ & 0.717 \\
\hline Hypertension & $4(21)$ & $1(20)$ & $2(100)$ & 0.053 \\
\hline Hyperlipidaemia & $2(11)$ & $1(20)$ & $1(50)$ & 0.322 \\
\hline Previous cholecystectomy & $3(16)$ & $1(20)$ & 0 & 0.799 \\
\hline \multicolumn{5}{|l|}{ Weight $(\mathrm{kg})$} \\
\hline Pre-operative & $114.8( \pm 17.6)$ & $111.6( \pm 12.2)$ & $131.9( \pm 11.9)$ & 0.338 \\
\hline Post-operative & $87.0( \pm 17.6)$ & $86.3( \pm 12.3)$ & $111.5( \pm 3.2)$ & 0.148 \\
\hline \multicolumn{5}{|l|}{ BMI $\left(\mathrm{kg} / \mathrm{m}^{2}\right)$} \\
\hline Pre-operative & $41.8( \pm 6.5)$ & $40.5( \pm 1.9)$ & $46.7( \pm 2.3)$ & 0.453 \\
\hline Post-operative & $31.7( \pm 5.7)$ & $31.5( \pm 3.5)$ & $40.1( \pm 0.6)$ & 0.117 \\
\hline \multicolumn{5}{|l|}{ Percentage body fat } \\
\hline Pre-operative & $48.2( \pm 4.9)$ & $50.5( \pm 0.8)$ & $59.5( \pm 8.7)$ & *0.013 \\
\hline Post-operative & $36.8( \pm 7.4)$ & $39.1( \pm 5.2)$ & $49.9( \pm 2.8)$ & 0.052 \\
\hline \% EWL-median (IQR) & $67.9(26.6)$ & $52.4(32.2)$ & 31.3 & $* 0.043$ \\
\hline
\end{tabular}

$R Y G B$ Roux-en-Y gastric bypass, $S G$ sleeve gastrectomy, $I G B$ intra-gastric balloon, $E W L$ excess weight loss, $B M I$ body mass index, $D M$ diabetes mellitus

*Denotes statistically significant difference between the treatment groups (Kruskal Wallis test) 
Table 2 Dietary intake

\begin{tabular}{llll}
\hline & $\begin{array}{l}\text { Pre-operative, } \\
N=26\end{array}$ & Post-operative, $N=26$ & $P$ value \\
\hline Energy (kcal/day) & $1947( \pm 669)$ & $1634( \pm 565)$ & $* 0.023$ \\
Fibre intake (g/day) & $24.4( \pm 12.1)$ & $17.5( \pm 7.3)$ & $* 0.008$ \\
Water intake (L/day) & $2.5( \pm 1.2)$ & $2.3( \pm 0.8)$ & 0.220 \\
Fat intake (g/day) & $64.3( \pm 25.8)$ & $59.2( \pm 22.3)$ & 0.301 \\
Calcium intake (mg/day) & $757( \pm 261)$ & $674( \pm 304)$ & 0.232 \\
\hline
\end{tabular}

*Denotes statistically significant difference between the pre- and post-operative groups

prospective study of a general population sample is shown for comparison [12]. At 6 months follow-up, 15 out of 26 patients had a lower usual BSFS compared with that pre-operatively, four had a higher score and there was no change in the remain$\operatorname{der}(P=0.028)$. Figure 2 shows the usual BSFS as analysed in four distinct groups. There is a statistically significant trend towards the lower ranked groups, i.e. firmer stools $(P=0.039)$.

Overall, there was a statistically significant reduction in the mean usual BSFS score (towards firmer stool) from $4.1( \pm 1.3)$ pre- to $3.1( \pm 1.9)$ post-operatively $(P=0.016)$. Figure 3 shows a statistically significant decrease in usual BSFS score in patients undergoing RYGB $(P=0.032)$, but not for $\mathrm{SG}$ $(P=0.176)$ or IGB $(P=0.655)$. There were no significant correlations between the change in BSFS and alimentary limb length (RYGB only), or any of the reported dietary intake measures.

\section{Constipation, Diarrhoea and Use of Medication}

At 6 months post-operatively, the proportion of patients suffering from constipation increased from 8 to $27 \%$, but this difference was not statistically significant $(P=0.125)$. The use of opiate analgesia did not significantly change pre- to postoperatively, 12 and $8 \%$, respectively $(P=0.317)$. The use of laxatives increased from $12 \%$ pre- to $19 \%$ post-operatively,

Fig. 1 Frequency of bowel motions pre-operatively and at 6 months post-operative followup but this difference was not statistically significant $(P=0.688$ ). None of the patients suffered from diarrhoea pre-operatively, and only one patient reported significant diarrhoea at followup. This patient was treated by IGB and concurrently commenced on Orlistat.

\section{Faecal Incontinence}

None of the participants reported faecal incontinence pre- or post-operatively. One participant reported frequent stool seepage/staining pre-operatively (five episodes after eight recorded bowel motions); however, this resolved post-operatively. No other participants reported stool seepage/staining. None of the participants reported use of pads pre-operatively; however, one participant did report using pads post-operatively. Faecal urgency, defined as the inability to postpone bowel motions by at least $15 \mathrm{~min}$, did not change significantly $(P=0.796)$.

\section{Discussion}

This study shows that constipation is a common problem after bariatric surgery with over a quarter of patients affected at 6 months follow-up. There was a significant $33 \%$ decrease

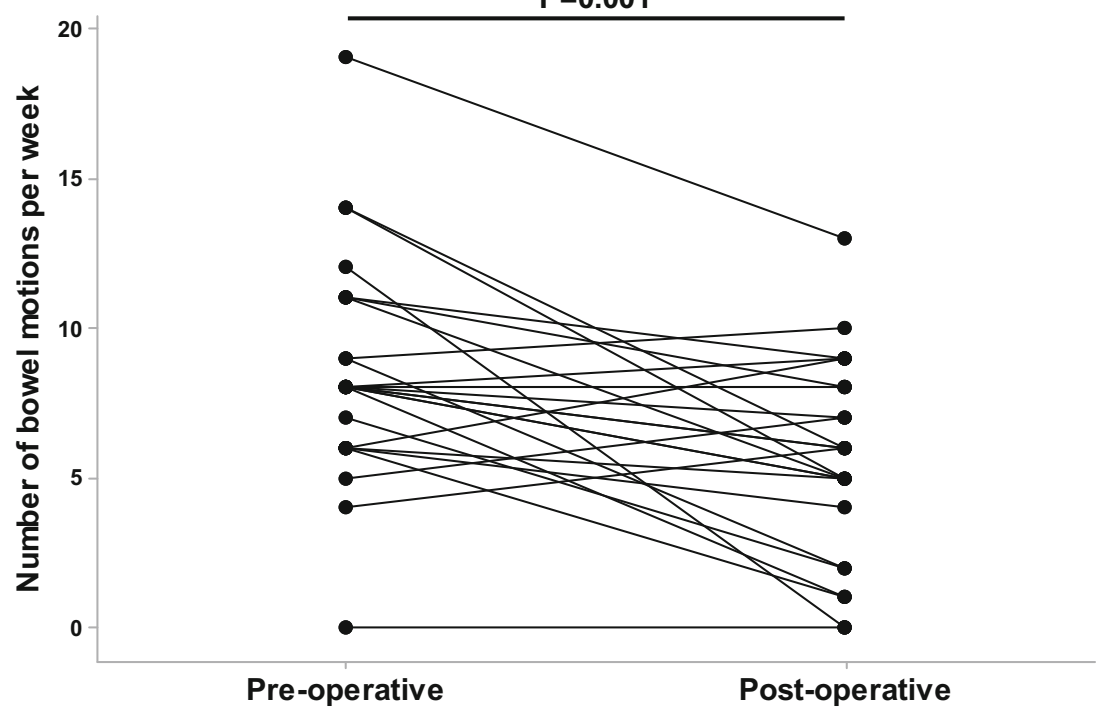


Table 3 Bristol Stool Form Scale in the present and a previous study

\begin{tabular}{|c|c|c|c|c|}
\hline Bristol Stool Form Scale & $\begin{array}{l}\text { Pre-operative } \\
N=26\end{array}$ & $\begin{array}{l}\text { Post-operative } \\
N=26\end{array}$ & $P$ value & $\begin{array}{l}\text { Heaton et al [12] } \\
N=1897\end{array}$ \\
\hline Not recorded & & & $P=0.028$ & \\
\hline$n(\%)$ & $1(3.8 \%)^{\mathrm{a}}$ & $3(11.5 \%)^{\mathrm{a}}$ & & - \\
\hline \multicolumn{5}{|c|}{ 1. Separate hard lumps, like nuts } \\
\hline$n(\%)$ & 0 & $3(11.5 \%)$ & & $95(8.1 \%)$ \\
\hline \multicolumn{5}{|c|}{ 2. Sausage-shaped but lumpy } \\
\hline$n(\%)$ & $2(7.7 \%)$ & $4(15.4 \%)$ & & $153(13.1 \%)$ \\
\hline \multicolumn{5}{|c|}{ 3. Like a sausage or snake but with cracks on its surface } \\
\hline$n(\%)$ & $1(3.8 \%)$ & $3(11.5 \%)$ & & $214(18.4 \%)$ \\
\hline \multicolumn{5}{|c|}{ 4. Like a sausage or snake, smooth and soft } \\
\hline$n(\%)$ & $12(46.2 \%)$ & $8(30.8 \%)$ & & $518(44.5 \%)$ \\
\hline \multicolumn{5}{|c|}{ 5. Soft blobs with clear-cut edges } \\
\hline$n(\%)$ & $8(30.8 \%)$ & $3(11.5 \%)$ & & $62(5.4 \%)$ \\
\hline \multicolumn{5}{|c|}{ 6. Fluffy pieces with ragged edges, a mushy stool } \\
\hline$n(\%)$ & $2(7.7 \%)$ & $1(3.8 \%)$ & & $53(4.5 \%)$ \\
\hline \multicolumn{5}{|l|}{ 7. Watery, no solid pieces } \\
\hline$n(\%)$ & 0.0 & $1(3.8 \%)$ & & Not assessed \\
\hline
\end{tabular}

${ }^{a}$ Three patients did not record any bowel motions post-operatively during the 7-day diary, one of whom returned a similar nil record pre-operatively. Discussion with the participants confirmed that these were accurate records in each case (i.e. no bowel motions during the 7 days). As such, this data is included and analysed as zero rather than being excluded from analysis

b Wilcoxon signed rank test

in frequency of bowel motions and a change towards firmer stools, suggesting a slowdown in gut transit post-operatively. This effect was observed despite a small non-significant increase in the use of laxatives, which is likely to be ameliorating the problem in those patients. Since dietary fibre intake has major roles in determining stool volume and gut transit times $[16,17]$, these changes may be explained, at least in

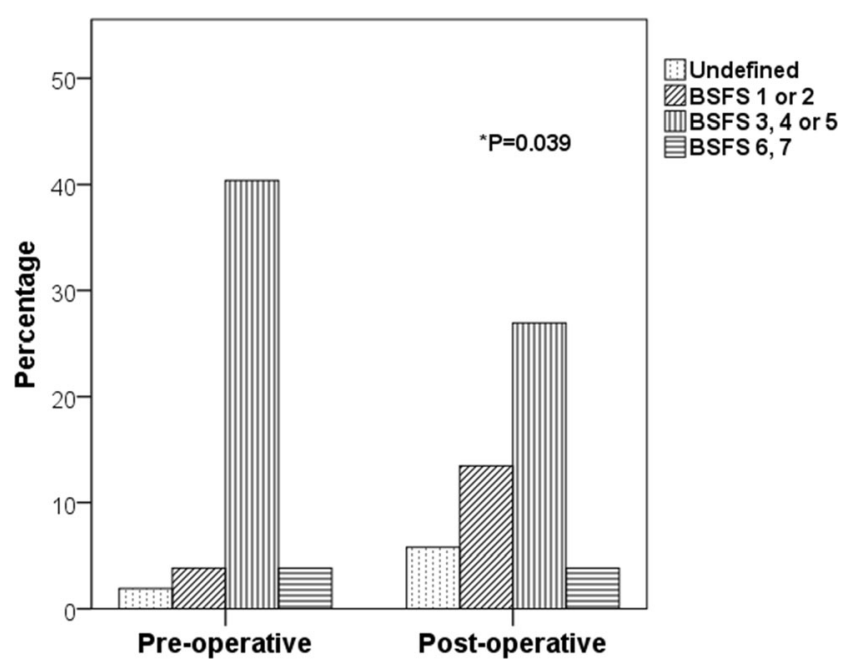

Fig. 2 Bristol Stool Form Scale groups before and after bariatric procedures part, by the $28 \%$ decrease in intake of dietary fibre we have observed after bariatric procedures. Only $15 \%$ of participants met the recent SACN recommendations of dietary fibre intake for adults post-operatively [15]. This could be due to difficulties in obtaining adequate fibre from the smaller food portions after bariatric procedures.

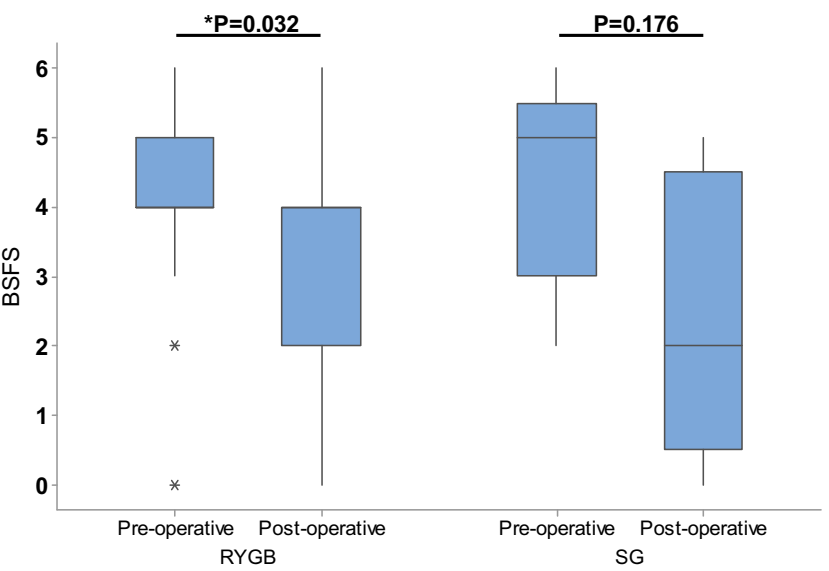

Fig. 3 Usual Bristol Stool Form Scale before and after the different bariatric procedures. IGB patients not shown $(n=2)$. Central line represents median (BSFS score 4 for RYGB both pre- and postoperatively). Top and bottom lines of box represent 75 th and 25th centiles, respectively. Whiskers represent range (minimum to maximum). *Represents outliers, defined as at least 1.5 times IQR away from 25 th or 75 th percentile. The $P$ value for RYGB remains significant after exclusions of outliers $(P=0.032)$ 


\section{Findings in the Context of Other Studies}

As highlighted by a systematic review, there is much heterogeneity in the published literature on the effects of bariatric surgery on bowel function [3]. Our finding of decreased stool frequency is in keeping with observations by Foster et al. [9].

In contrast to our findings, Potoczna et al. [7] described a decrease in constipation from $29.4 \%$ pre- to $7.1 \%$ preoperatively $(P<0.001)$. They also found an increase in the frequency of loose stools (6.3 to $40.5 \%$ ) and diarrhoea (1.6 to $5.6 \%$ ). In their study, subjects had a $250-\mathrm{cm}$ alimentary limb compared with a mean of $124 \mathrm{~cm}$ in the present study. This difference in surgical technique is likely to be a major factor in explaining the difference in findings. Longer alimentary limbs have been associated with an increase in malabsorptive complications, including an increase in diarrhoea symptoms [18]. The limb lengths used in our RYGB technique are unlikely to cause malabsorption [19]. This is supported by the low incidence of post-operative diarrhoea in this cohort.

Furthermore, the timing of assessment of bowel habit after bariatric surgery may be an important factor. Potoczna et al. assessed RYGB patients at a median of 2.1 years (range 4 months to 5.4 years) post-operatively compared to 6.4 months in the present study. However, given the wide range of followup periods in the study by Potoczna et al., the authors analysed the impact of follow-up time on bowel habit and concluded that no significant adaptation was occurring over time.

Our finding of lower BSFS post-operatively suggests an increase in WGTT [11]. This effect may be in part due to the increased post-prandial levels of glucagon-like peptide-1 (GLP-1) and peptide-YY (PYY) after bariatric surgery which delays intestinal transit [20-22]. However, an increase in gastric emptying as well as a faster oro-caecal transit time after RYGB have been reported by some [23, 24], whereas others have not found any increase in either gastric emptying [25, 26] or gut transit time [24]. Fibre has an acceleratory effect on transit time [17], and our findings suggestive of an increase in WGTT post-operatively are consistent with the observed fall in fibre intake.

Bariatric procedures can result in vagal nerve injury [27, 28]. The now seldom used total and selective vagotomy caused diarrhoea in a subset of patients [29]. Our RYGB technique involved mobilising the stomach by dissection of the gastrohepatic ligament close to the gastric wall. This preserves the main vagal trunks in a fashion similar to a highly selective vagotomy and makes extensive vagal nerve injury unlikely. This is consistent with the very low incidence of postoperative diarrhoea in this cohort.

\section{Strengths and Limitations of Study}

The small sample size and the use of self-reported data are the limitations of this study. The reliability of self-reported data is a recognised problem in this patient population [30, 31]. In addition, we collected follow-up data at 6 months post-operatively, and dietary adaptation following RYGB is thought to be occurring from 3 months to at least 2.5 years post-surgery [32]. So, the changes which we observed may not represent a new steady state for these patients. Repeated assessments over longer-term follow-up periods would be useful.

The use of a prospective study design with paired observations using validated assessment tools is the major strength of this study. In particular, the availability of data on individual dietary intakes obtained using a validated FFQ is valuable in developing potential explanations for the observed effects on bowel habit. The observed decrease in dietary fibre intake offers a very practical target for interventions to address the observed adverse effects on bowel habit.

\section{Conclusions}

In summary, in obese patients following bariatric surgery, we found decreased bowel motion frequency and reduced BSFS, which are indicative of prolonged intestinal transit time. These observations were associated with reduced dietary fibre intake which is a likely causal relationship. These findings should alert healthcare professionals involved in caring for obese patients undergoing bariatric procedures to this potentially treatable post-operative problem. Future studies into the optimal dietary advice, treatment and possible fibre supplementation in this group are needed.

\section{Compliance With Ethical Standards}

Conflict of Interest The authors declare that they have no conflict of interest.

Ethical Approval The study was approved by the Newcastle \& North Tyneside National Research Ethics Service Committee (13/NE/0204). All procedures performed in the study were in accordance with the ethical standards of the institutional and/or national research committee and with the 1964 Helsinki declaration and its later amendments or comparable ethical standards.

Open Access This article is distributed under the terms of the Creative Commons Attribution 4.0 International License (http:// creativecommons.org/licenses/by/4.0/), which permits unrestricted use, distribution, and reproduction in any medium, provided you give appropriate credit to the original author(s) and the source, provide a link to the Creative Commons license, and indicate if changes were made.

\section{References}

1. Aro P, Ronkainen J, Talley NJ, et al. Body mass index and chronic unexplained gastrointestinal symptoms: an adult endoscopic population based study. Gut. 2005;54(10):1377-83. 
2. Sjostrom L. Review of the key results from the Swedish Obese Subjects (SOS) trial - a prospective controlled intervention study of bariatric surgery. J Intern Med. 2013;273(3):219-34.

3. Poylin V, Serrot FJ, Madoff RD, et al. Obesity and bariatric surgery: a systematic review of associations with defecatory dysfunction. Color Dis: Off J Assoc Coloproctol Great Britain Ireland. 2011;13(6):e92-e103.

4. Belsey J, Greenfield S, Candy D, et al. Systematic review: impact of constipation on quality of life in adults and children. Aliment Pharmacol Ther. 2010;31(9):938-49.

5. Monnikes H. Quality of life in patients with irritable bowel syndrome. J Clin Gastroenterol. 2011;45(Suppl):S98-S101.

6. Bouchoucha M, Fysekidis M, Julia C, Airinei G, Catheline JM, Reach G, et al. Functional Gastrointestinal Disorders in Obese Patients. The Importance of the Enrollment Source. Obesity surgery. 2015.

7. Potoczna N, Harfmann S, Steffen R, et al. Bowel habits after bariatric surgery. Obes Surg. 2008;18(10):1287-96.

8. El Labban S, Safadi B, Olabi A. The Effect of Roux-en-Y Gastric Bypass and Sleeve Gastrectomy Surgery on Dietary Intake, Food Preferences, and Gastrointestinal Symptoms in Post-Surgical Morbidly Obese Lebanese Subjects: a Cross-Sectional Pilot Study. Obes Surg. 2015.

9. Foster A, Laws HL, Gonzalez QH, et al. Gastrointestinal symptomatic outcome after laparoscopic Roux-en-Y gastric bypass. J Gastrointest Surg : Off J Soc Surg Aliment Tract. 2003;7(6):750-3.

10. Degen LP, Phillips SF. How well does stool form reflect colonic transit? Gut. 1996;39(1):109-13.

11. Lewis SJ, Heaton KW. Stool form scale as a useful guide to intestinal transit time. Scand J Gastroenterol. 1997;32(9):920-4.

12. Heaton KW, Radvan J, Cripps H, et al. Defecation frequency and timing, and stool form in the general population: a prospective study. Gut. 1992;33(6):818-24.

13. Drossman DA. Rome III: the new criteria. Chin J Dig Dis. 2006;7(4):181-5.

14. Bingham SA, Gill C, Welch A, et al. Validation of dietary assessment methods in the UK arm of EPIC using weighed records, and 24-hour urinary nitrogen and potassium and serum vitamin $C$ and carotenoids as biomarkers. Int J Epidemiol. 1997;26 Suppl 1:S13751.

15. SACN Carbohydrates and Health Report. In: England PH, editor. 2015.

16. Cummings JH, Stephen AM. Carbohydrate terminology and classification. Eur J Clin Nutr. 2007;61 Suppl 1:S5-S18.
17. Eswaran S, Muir J, Chey WD. Fiber and functional gastrointestinal disorders. Am J Gastroenterol. 2013;108(5):718-27.

18. Freeman JB, Kotlarewsky M, Phoenix C. Weight loss after extended gastric bypass. Obes Surg. 1997;7(4):337-44.

19. Stefanidis D, Kuwada TS, Gersin KS. The importance of the length of the limbs for gastric bypass patients - an evidence-based review. Obes Surg. 2011;21(1):119-24.

20. Flint A, Raben A, Ersboll AK, et al. The effect of physiological levels of glucagon-like peptide-1 on appetite, gastric emptying, energy and substrate metabolism in obesity. Int J Obes Relat Metab Disord : J Int Assoc Study Obes. 2001;25(6):781-92.

21. Savage AP, Adrian TE, Carolan G, et al. Effects of peptide YY (PYY) on mouth to caecum intestinal transit time and on the rate of gastric emptying in healthy volunteers. Gut. 1987;28(2):166-70.

22. Roux CW, Aylwin SJB, Batterham RL, et al. Gut hormone profiles following bariatric surgery favor an anorectic state, facilitate weight loss, and improve metabolic parameters. Ann Surg. 2006;243(1): 108-14.

23. Morinigo R, Moize V, Musri M, et al. Glucagon-like peptide-1, peptide $\mathrm{YY}$, hunger, and satiety after gastric bypass surgery in morbidly obese subjects. J Clin Endocrinol Metab. 2006;91(5):173540.

24. Hedberg J, Hedenstrom H, Karlsson FA, et al. Gastric emptying and postprandial PYY response after biliopancreatic diversion with duodenal switch. Obes Surg. 2011;21(5):609-15.

25. Naslund I, Beckman KW. Gastric emptying rate after gastric bypass and gastroplasty. Scand J Gastroenterol. 1987;22(2):193-201.

26. Horowitz M, Cook DJ, Collins PJ, et al. Measurement of gastric emptying after gastric bypass surgery using radionuclides. Br J Surg. 1982;69(11):655-7.

27. Ballsmider LA, Vaughn AC, David M, et al. Sleeve gastrectomy and Roux-en-Y gastric bypass alter the gut-brain communication. Neural Plast. 2015;2015:601985.

28. Berthoud HR, Shin AC, Zheng H. Obesity surgery and gut-brain communication. Physiol Behav. 2011;105(1):106-19.

29. George JD, Magowan J. Diarrhea after total and selective vagotomy. Am J Dig Dis. 1971;16(7):635-40.

30. Lichtman SW, Pisarska K, Berman ER, et al. Discrepancy between self-reported and actual caloric intake and exercise in obese subjects. N Engl J Med. 1992;327(27):1893-8.

31. Schoeller DA. Limitations in the assessment of dietary energy intake by self-report. Metab Clin Exp. 1995;44(2 Suppl 2):18-22.

32. Thomas JR, Marcus E. High and low fat food selection with reported frequency intolerance following Roux-en-Y gastric bypass. Obes Surg. 2008;18(3):282-7. 